\title{
Influence of Oleic and Lauric Acid on the Stability of Magnetorheological Fluids
}

\author{
Yuehua Huang ${ }^{1}$, Yuhuan Jiang ${ }^{1}$, Xiongbo Yang ${ }^{2 *}$, and Ruizhen $\mathrm{Xu}^{2}$ \\ ${ }^{I}$ College of Electrical Engineering \& New Energy, China Three Gorges University, Yichang, China \\ ${ }^{2}$ College of Science, China Three Gorges University, Yichang, China
}

(Received 11 May 2015, Received in final form 7 July 2015, Accepted 13 August 2015)

\begin{abstract}
In this paper, magnetorheological fluids (MRFs) based on micro-sized iron particles dispersed in silicone oil are presented. The iron particles are modified by adding different ratios of oleic acid and lauric acid as surfactants to the suspensions. Lauric acid was found to reduce the stability of the MRFs, and more lauric acid results in a higher rate of sedimentation. Further study showed that the formation and structure of lauric acid may result in the sedimentation of micrometer-sized particles. Meanwhile, the electro-resistance of MRF in this paper shows a decrease from beyond $6000 \mathrm{M} \Omega$ to $190 \Omega$ with an increase in the external field from $0 \mathrm{mT}$ to $400 \mathrm{mT}$.
\end{abstract}

Keywords : MRF, stability, oleic acid, lauric acid, electrical properties

\section{Introduction}

Magnetorheological fluids (MRFs) are a new type of controllable, intelligent materials [1] that typically consist of ferro- or ferri-magnetic microparticles (e.g., iron and iron oxide, etc.) suspended and dispersed in a liquid matrix that contains a carrier liquid (e.g., mineral oil, silicone oil or water), surfactants and additives $[2,3]$. The microparticles in MRFs are known to have the ability to form chains when an external magnetic field is applied [4]. The ordering of the particles, which influences the rheological and electrical properties of the material, depends on the intensity of the magnetic field that is applied [5, 6]. For example, MRFs have already been applied in dampers, brakes, journal bearings, pneumatic artificial muscles, optics finishing, fluid clutches, and aerospace components, as a result of their particular rheological properties (e.g., viscosity) [7-9].

However, the gravity and agglomeration of magnetic particles may lead to instability in the MRFs [10]. It is difficult to prevent sedimentation in MRFs, and great efforts have been made to this end [12]. In general, surfactants and additives have been introduced to reduce particle sedimentation and to improve the stability of the MRFs [13-16]. For instance, different annexing agents,

(C)The Korean Magnetics Society. All rights reserved.

*Corresponding author: Tel: +86-13545836571

Fax: +86-717-6392618, e-mail: yuhuanjiang1990@163.com such as lauric acid, stearic acid, oleic acid and sodium dodecyl benzene sulfonate, have been introduced to improve the hydrophobic property of the surface of the particles, and particles coated with a polymer have been observed to have a reduced density [17, 18]. Agglomeration can be reduced when oleic acid molecules are coated on the magnetic particles, and lauric acid has an advantage in that it forms a kind of flocculent structure within the system to prevent sedimentation. In this work, a combination of oleic acid and lauric acid is considered to modify the surface of the magnetic particles to ensure these are sufficiently dispersed in a carrier liquid consisting of silicone oil. The influence of the oleic acid and lauric acid on the stability of the magnetorheological fluids is investigated by analyzing the sedimentation indifferent samples.

\section{Experimental Methods}

\subsection{Production of MRFs}

The MRFs in this work were obtained by mixing carbonyl iron particles, silicone oil, stearic acid and lauric acid. The particles in carbonyl iron powder have a mean diameter of 6 to 8 microns. Fig. 1 shows an SEM image of the carbonyl iron particles. The setup that was used to produce the MRFs is shown in Fig. 2. The system mainly consists of an electric stirrer, an electric heater, and a three-neck flask.

The MRFs were prepared according to the following 


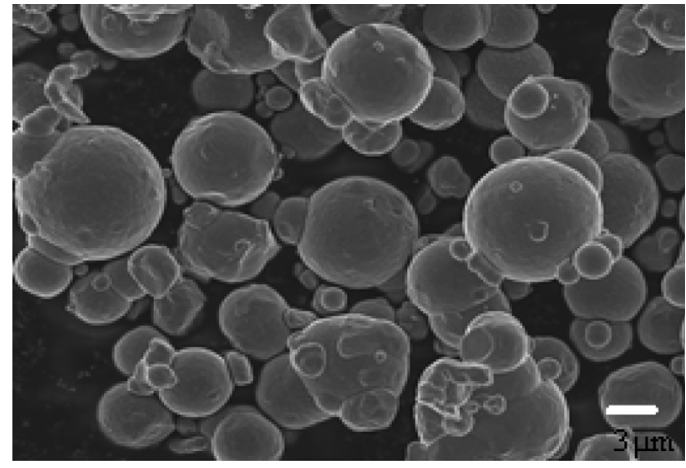

Fig. 1. SEM image of the carbonyl iron particles.

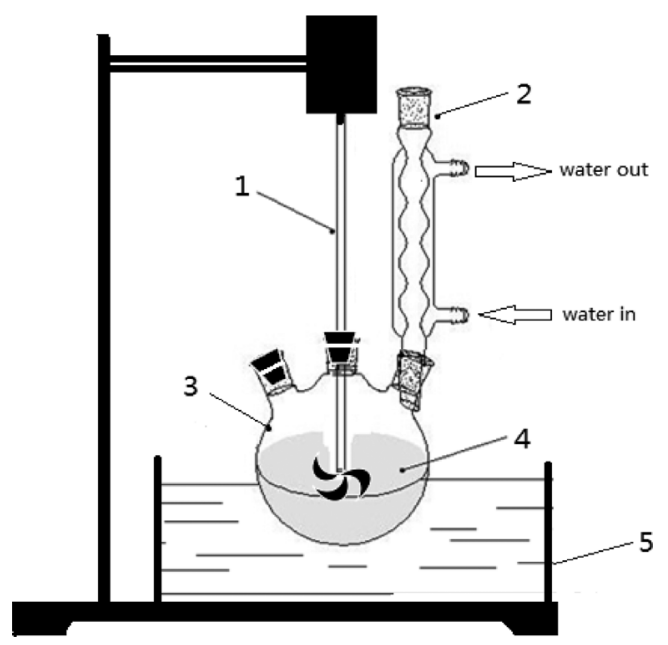

Fig. 2. Experimental installation for the MRF production: (1) $100-\mathrm{W}$ electric stirrer; (2) condenser, (3) three-neck flask, (4) liquid suspension, (5) 300-W electric-heated water bath.

procedures:

Step 1: Surface modification of the iron particles.

The oleic acid and iron powder are mixed with an anhydrous ethanol solvent in a flask, and the mixture is strenuously stirred using a mechanical agitator at $40^{\circ} \mathrm{C}$. After several hours, the extra solvent is evaporated to obtain iron particles with hydrophobic surfaces.

Step 2: Preparation of the suspension.

A suitable amount of modified iron particles are stirred with silicone oil and lauric acid.

This paper focuses on the effect that the lauric acid and oleic acid have on the stability of the MRFs. Four groupcomparable tests were designed, as shown in Table 1. $\alpha$ represents the mass ratio of oleic acid and carbonyl iron powder. $\beta$ represents the mass score of the lauric acid. A, $\mathrm{B}, \mathrm{C}$, and D represent the four groups of tests with different $\alpha$. In each group, the mass score of the oleic acid is kept to at the same value for group A of 0.2, group $\mathrm{B}$ of 0.5 , group C $0 \mathrm{f} 0.7$ and group D of 1.0. Every group
Table 1. Parameters for the MRF samples.

\begin{tabular}{cccc}
\hline \hline Sample & $\begin{array}{c}\text { Mass ratio of oleic } \\
\text { acid and carbonyl } \\
\text { iron powder } \alpha\end{array}$ & $\begin{array}{c}\text { Mass score of } \\
\text { lauric acid } \\
\beta(\%)\end{array}$ & $\begin{array}{c}\text { Mass score of } \\
\text { carbonyl iron powder } \\
\phi(\%)\end{array}$ \\
\hline A1 & 0.2 & 0 & 50 \\
A2 & 0.2 & 1 & 50 \\
A3 & 0.2 & 5 & 50 \\
A4 & 0.2 & 7 & 50 \\
B1 & 0.5 & 0 & 50 \\
B2 & 0.5 & 1 & 50 \\
B3 & 0.5 & 5 & 50 \\
B4 & 0.5 & 7 & 50 \\
C1 & 0.7 & 0 & 50 \\
C2 & 0.7 & 1 & 50 \\
C3 & 0.7 & 5 & 50 \\
C4 & 0.7 & 7 & 50 \\
D1 & 1.0 & 0 & 50 \\
D2 & 1.0 & 1 & 50 \\
D3 & 1.0 & 5 & 50 \\
D4 & 1.0 & 7 & 50 \\
\hline
\end{tabular}

has four different tests, and the mass score for lauric acid added to MRF is $0 \%, 1 \%, 5 \%, 7 \%$, respectively. In total, 16 different tests were carried out, as listed in Table 1.

\subsection{Experiment}

Sedimentation rate was measured by conducting visual observation of the position of the boundary between the clear and turbid parts of the carrier oil. The prepared samples were placed into cylindrical glass test tubes (diameter $15 \mathrm{~mm}$ ), and the sedimentation ratio $\varphi$ is simply defined as the proportion of the length between the clear and the turbid parts of the MRFs.

$$
\varphi=1-\frac{h_{2}}{h_{1}}
$$

where $h_{1}$ is the initial length of the MRFs, and $h_{2}$ is the length of the turbid part. The data for $h_{2}$ was recorded every hour until the MRFs were stable. The sedimentation ratios were compared between the different samples to study the influence that the oleic acid and the lauric acid had on the stability of the MRFs.

The resistance of the MRF was recorded by constructing an MRF resistor consisting of a glass cube containing MRF and two electrodes at the two opposite sides. The MRF resistor is placed in a controllable external field, and an ohm gauge was used to measure the resistance.

\section{Results and Discussion}

\subsection{Influence of lauric acid on the stability of MRFs}



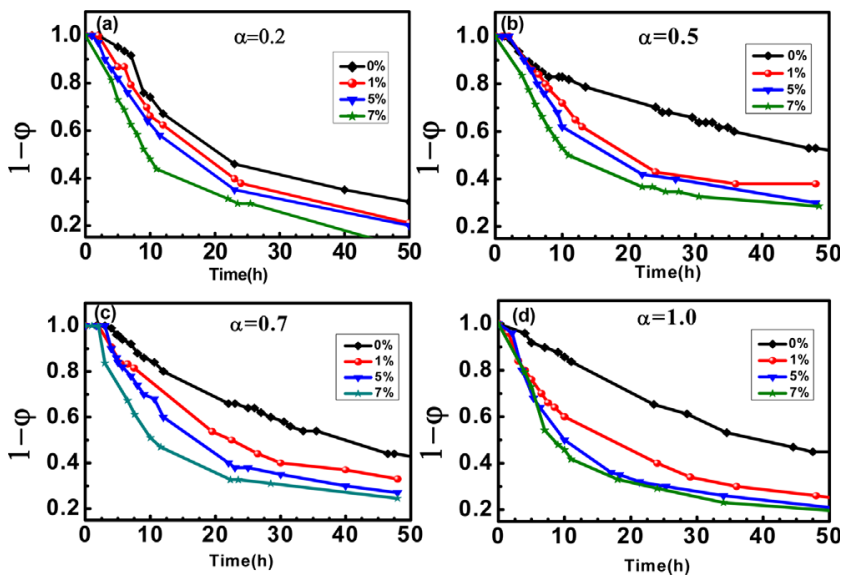

Fig. 3. (Color online) Variation in $1-\varphi$ according to time for the MRFs (a) in group A, (b) in group B, (c) in group C, (d) in group $\mathrm{D}$.

The four groups of experiments with 16 different samples are listed in Table 1 to distinguish the influence that the lauric acid had on the sedimentation rate of the suspension. The MRF samples are placed into cylindrical glass test tubes, for which $h_{1}$ is the initial length of the MRFs, and $h_{2}$ is the length of the turbid part during the standing process.

Fig. 3 shows time-settlement curves for the MRFs. The increase in $\beta$ is associated with a decrease in the $1-\varphi$ value that indicated a faster sedimentation rate in each group. This means that more lauric acid leads to worse stability of the MRFs, which is inconsistent with the fact that lauric acid is an active surface agent that could help prevent sedimentation in MRF. Therefore, the experimental results prove that the addition of lauric acid may not always be an effective solution.

The phenomenon is a result of the molecular structures of lauric acid and oleic acid and the preparation technology that is used. The oleic acid and lauric acid molecules have similar structures, as shown in Fig. 4, and the chain structure has a hydrophilic group and a lipophilic group on both sides of it. During preparation, when the carbonyl iron powder is surface-modified with oleic acid, the hydrophilic group of oleic acid molecules first cover the surface of the iron molecules, and this prevents the lauric acid molecules from making contact with the iron. The lipophilic group of lauric acid connects to the lipophilic group of oleic acid, and hydrophilic groups are left, which is exclusive to silicone oil molecules. A repulsive force between the hydrophilic groups and the lipophilic groups causes a reduction in the stability of the suspensions and a faster sedimentation. However, the mass ratio $\alpha$ plays an important role during preparation. In this paper, tests are

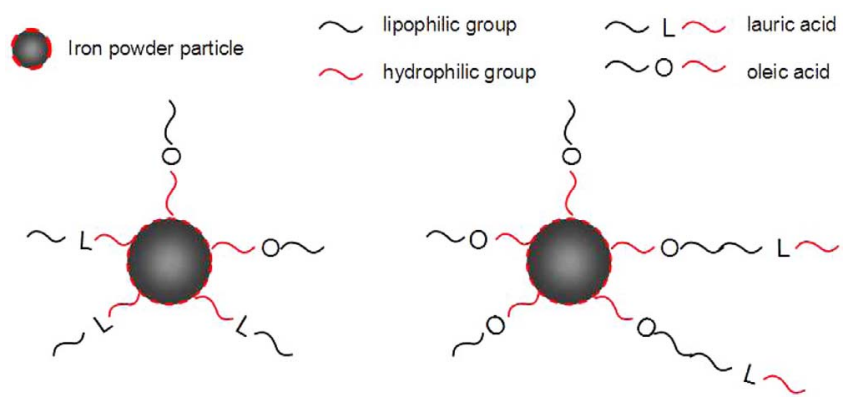

Fig. 4. (Color online) Illustration of the molecules combined with the iron particles.

only performed when the value of $\alpha$ is beyond 0.2 , and the further addition of oleic acid results in fewer opportunities for lauric acid molecules to make contact with the iron particles.

\subsection{The influence of oleic acid on the stability of MRFs}

Fig. 3 indicates that suspensions without lauric acid are much more stable, so suspensions without lauric acid have been selected as the samples to investigate the relationship between the presence of oleic acid and stability. Five comparable tests were made where the value of $\beta$ remains as zero while the value of $\alpha$ varies
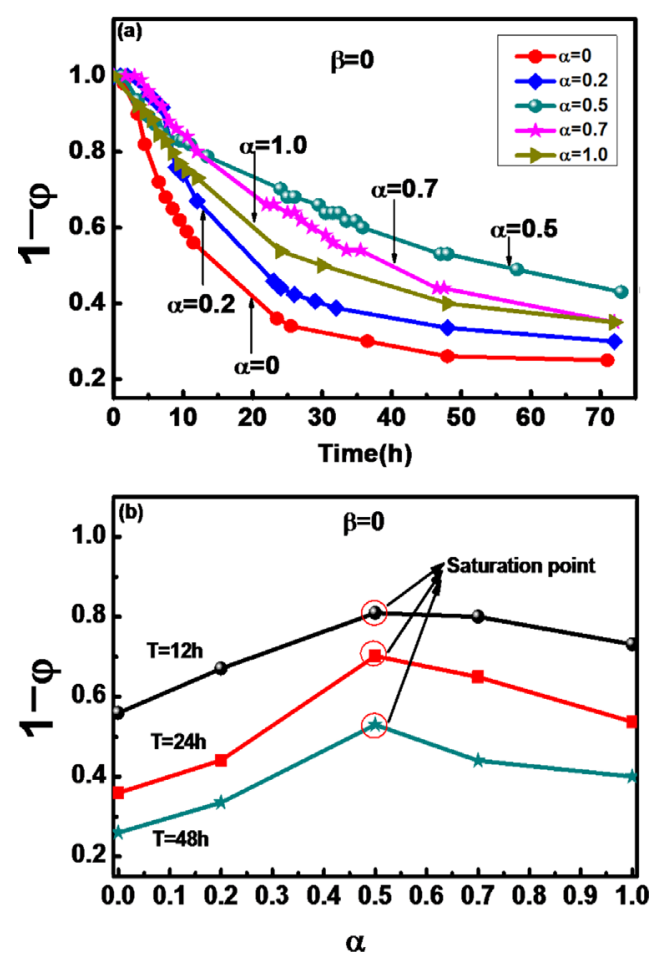

Fig. 5. (Color online) (a) variation in $1-\varphi$ with respect to time for MRF under different $\alpha$; (b) variation of $1-\varphi$ by $\alpha$ under the same $\beta$ and time. 
from 0 to $0.2,0.5,0.7$, and 1.0 , respectively. Fig. 5(a) shows the time-settlement curves of the suspensions without lauric acid. The iron particles are modified by the oleic acid during preparation to be well mixed with silicone oil.

The Fig. 5(a) shows how the curves are less steep as $\alpha$ increases when $\alpha$ is less than 0.5 . This means that more oleic acid produces a suspension that is more stable. Meanwhile, when $\alpha$ is over 0.5 , the suspension becomes less stable since the excess of oleic acid leads to an excess in hydrophilic groups that repulse the silicone oil molecules. Fig. 5(b) provides the $\alpha$-settlement curves for three different time periods, which make it more clear to observe the variation in the trend for the samples at different $\alpha$. This shows that $\alpha=0.5$ is the saturation point, which is in accordance with the changing law in Fig. 5 (a). It is important to note that when the mass ratio $\alpha$ is $0.5,1-\varphi$ reaches its peak value, and the suspension achieves the highest stability when the mass ratio of the oleic acid and carbonyl iron powder is 0.5 .

\section{Electrical Properties of MRF}

The MRFs obtained from carbonyl iron powder in silicone oil with oleic acid become electro-conductive in a magnetic field due to the formation of conductive chains. The electrical resistance for sample A1 in an alternating magnetic field was analyzed, and the A1 MRF exhibited a very low resistance under the magnetic field. The variation in $\mathrm{R}$ under different magnetic field is recorded in Fig. 6. In an absence of a field, the resistance of $\mathrm{R}$ increased beyond $6 \times 10^{9} \Omega$, and when a field was applied an increased to $400 \mathrm{mT}$, the large resistance of the MRF decreased to $190 \Omega$, which indicates it is possible to control the characteristics of the suspension from an insulator to a conductor.

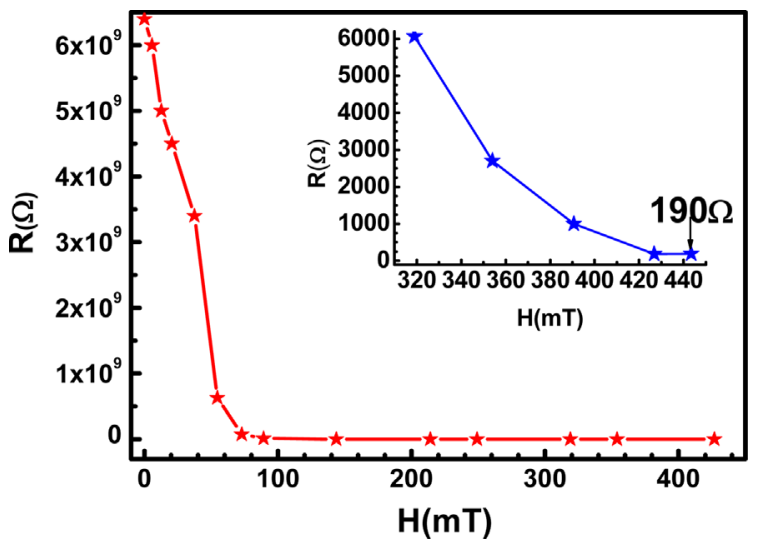

Fig. 6. (Color online) Variation in R for MRF with an increasing external field.
In the absence of a magnetic field, the magnetic particles in the carrier liquid are dispersed and disordered, and the intervals between the molecules are relatively large. Once an external magnetic field is applied to the magnetorheological fluids, the magnetic particles are brought together under the magnetic force, and flux linkages are formed one by one as if they were electric wires. The MRF changes from an insulator into a conductor under the changing magnetic field, so the resistance decreases from beyond $6 \times 10^{9} \Omega$ to $190 \Omega$.

\section{Conclusions}

In this paper, we have demonstrated the significant effect that lauric acid and oleic acid have on the stability and settling behavior of the given suspensions. The results suggest that the an increase in lauric acid leads to a worsening stability of the suspensions when $\alpha$ is fixed. This indicates that the formation and structure of lauric acid may cause sedimentation of the micrometer-sized particles. Furthermore, samples without lauric acid were selected to produce time settlement curves, and an adequate proportion of oleic acid was observed to result in a good stability. Furthermore, we select the MFR A1 sample to analyze the variation in the electrical resistances in an alternating magnetic field. $\mathrm{R}$ was observed to decrease from beyond $6 \times 10^{9} \Omega$ to when the external field increased from $0 \mathrm{mT}$ to $400 \mathrm{mT}$. These results thus provide guidance for new electrical applications of MRFs.

\section{Acknowledgements}

This work is financially supported by the Three Gorges University Graduate Student Innovation Fund (2014CX040), the Three Gorges University Master's Degree Thesis Pew Fund (2015PY033), the National Science Foundation of China (Grant No. 51177088), Key Scientific Research Project of Hubei Provincial Department of Education of China (No. D20151205) and Guiding Scientific Research Project of Hubei Provincial Department of Education of China (No. B2015246).

\section{References}

[1] J. W. Tu, Y. Yu, L. Huang, B. Tu, and J. Y. Xu, Mater. Sci. Technol. 18, 243 (2014).

[2] J. D. Carlson, and M. R. Jolly, Mechatronics, 10, 4 (2000).

[3] Ioan Bica, J. Ind. Eng. Chem. 13, 299 (2007).

[4] Ioan Bica, J. Ind. Eng. Chem. 12, 620 (2006).

[5] S. J. Dyke, B. F. Spencer, M. K. Sain, and J. D. Carlson, Mater. Struct. 5, 693 (1998). 
[6] X. Huang, A. Mohla, W. Hong, A. F. Bastawros, and X.Q. Feng, Soft Matter. 10, 1537 (2014).

[7] Y. Shiao, Q.-A. Nguyen, and G.-D. Huang, Mater. Sci. Technol. 18, 16 (2014).

[8] J. P. Rich, Patrick S. Doyle, Gareth H. Mckinley, Rheologica Acta 51, 1 (2012).

[9] Muhammad Aslam, Yao Xiong-liang, and Deng Zhongchao, J. Marine Sci. Appl. 5, 17 (2006).

[10] J. L. Viota, F. González-Caballero, J. D. G. Durán, and A. V. Delgado, J. Colloid Interface Sci. 309, 135 (2007).

[11] M. A. Willard, L. K. Kuriharal, E. E. Carpenter, S. Calvin, and V. G. Harris, Int. Mater. Rev. 49, 125 (2004).

[12] M. T. López-López, and J. de Vicente, J. Mater. Res. 20,
874 (2005).

[13] J. H. Park, M. H. Kwon, and O. Ok. Park, Chem. Eng. 18, 580 (2001).

[14] B. D. Chin, J. H. Park, M. H. Kwon, Rheol. Acta 40, 211 (2001).

[15] B. J. Park, C. W. Park, and S. W. Yang, J. Physics: Conference Series 149, 1 (2009).

[16] J. Park, H. H. Song, and H. J. Choi, Mater. Lett. 63, 1350 (2009).

[17] Doina Bica, Ladislau Vékás, Mikhail V. Avdeev, Oana Marinică, Vlad Socoliuc, Maria Bălăsoiu, Vasil M. Garamus, J. Magn. Magn. Mater. 311, 17 (2007). 